\title{
Entlarvung des Antisemitismus der Regierungen Vargas und Dutra. Über das Buch „Weltbürger - Brasilien und die jüdischen Flüchtlinge 1933-1948" von Maria Luiza Tucci Carneiro
}

[The Unmasking of Antisemitism of the Vargas and Dutra Governments. On the publication "Weltbürger - Brazil and the Jewish Refugees 1933-1948" by Maria Luiza Tucci Carneiro]

http://dx.doi.org/10.1590/1982-8837225228

Dieter Strauss ${ }^{1}$

CARNEIRO, Maria Luiza Tucci. Weltbürger-Brasilien und die jüdischen Flüchtlinge 19331948. Zürich und Berlin: Lit Verlag 2014. (477S.)

Nach außen sollte der Schein gewahrt werden: Demokratisch und für Flüchtlinge offen sollte Brasilien unter Vargas und Dutra erscheinen. Und einige europäische Zeitungen fielen auch darauf rein, wie zum Beispiel das schwedische „Socialdemocraten Folkets dagblad“, das im August 1939 in einem Leitartikel Vargas als Bekämpfer der NSExpansion, als neuen Bolivar feierte. (Vgl. CARNEIRO 2014: 44). Grund für die tolerante und humanitäre Maske war nach Maria Luiza Tucci CARNEIRO vor allem der Druck der USA. Die guten Wirtschaftsbeziehungen zu den Staaten wollte man nicht verlieren. (ebd.: 49; 415)

Der schöne Schein wurde aber auch von Stefan Zweig unterstützt, der Brasilien bereits im Herbst 1936 in seiner „Kleinen Reise nach Brasilien“ große Toleranz gegenüber der Rassenfrage bescheinigte. ${ }^{2}$ Und das, obwohl die deutsche Jüdin Olga Benario, die im November 1935 an dem gescheiterten kommunistischen Putsch gegen die Regierung Vargas teilgenommen hatte, damals als Schwangere gegen die

\footnotetext{
${ }^{1}$ Sachbuchautor und Referent in München; der Autor ist mit Anita L. Prestes Mitherausgeber des Buches Não olhe nos olhos do inimigo. Rio de Janeiro: Paz e Terra, 1995. Email: Straussda4@ yahoo.de

${ }^{2}$ Vgl. dazu Friederike Manthey, Reise ins Exil. Veröffentlichung „Casa Stefan Zweig“, 6.11.2010. Für F. Manthey bereitet die „Kleine Reise“ Zweigs „Brasilien: Ein Land der Zukunft“ von 1941 vor.
} 
brasilianische Verfassung an Hitler-Deutschland ausgeliefert wurde. Für Tucci Carneiro ein klarer Beweis, dass das Vargas Regime mit den Nazis gemeinsame Sache machte (Vgl. CARneIRo 2914: 22). Kein Hinderungsgrund für die deutsche Bundesrepublik, Vargas 1953 das Bundesverdienstkreuz zu verleihen, wie so vielen Ex-Nazis bzw. NSSympathisanten. Olga Benario konnte im Berliner Frauengefängnis in der Barnimstraße ihr Kind Anita noch zur Welt bringen und rund ein Jahr stillen. Dann wurde sie in die Konzentrationslager Lichtenberg und Ravensbrück deportiert und im Frühjahr 1942 in der Gaskammer von Bernburg ermordet. Anita wurde auf internationalen Druck an ihre brasilianische Großmutter übergeben. Sie lebt heute als Zeithistorikerin in Rio de Janeiro und hat in unserem Sammelband über Olga Benario Não olhe nos olhos do inimigo (vgl. PRESTES/STRAUSS 1995: 13ff) einen Artikel über ihre Mutter geschrieben.

Wenn schon bei der Abschiebung die Verfassung gebrochen wurde, wie steinig war dann der Weg für die vielen Juden, die nach Brasilien flüchten wollten? Genau darum geht es zentral in diesem Buch: Als die Nationalsozialisten den deutschen Juden ihre Staatsangehörigkeit aberkannten und diese als staatenlos galten, blies das Vargas Regime in dieses Horn und erklärte die Juden für nicht „,assimilierbar“. Sie waren eben „subversive oder zersetzende Elemente, die dazu neigten, rassische Enklaven, wahre Fremdkörper im nationalen Organismus“, zu bilden“ (CARNEIRO 2014: 50). Bei der amerikanophilen Haltung der Vargas Regierung wurden dann auch die Rundschreiben, die die Einreise regelten, ab 1937 als geheim eingestuft. Allen voran das geheime Rundschreiben Nr. 1.127, das die Visaerteilung an Juden untersagte und von Vargas und dem Arbeits-, Industrie- und Handelsministerium genehmigt worden war. Die Begründung lag wieder in der Reinerhaltung der brasilianischen Rasse. Darauf macht das Buch ebenso aufmerksam wie darauf, dass die brasilianische Regierung alle weiteren restriktiven Maßnahmen in der Einwanderungspolitik geheim hielt (ebd.: 91; 416). Mehr noch, die wenigen Diplomaten, die versuchten Juden Visa zu erteilen, wurden mit Verfahren verfolgt und angeklagt. Wie zum Beispiel der brasilianische Botschafter in Frankreich Luiz Martins de Souza Dantas (ebd.: 49).

Die Konferenz im französischen Évian am Genfer See hatte im Juli 1938 die Aufgabe, eine Lösung für die ausgebürgerten Flüchtlinge aus Deutschland und Österreich zu finden und zwar für Juden und Nichtjuden. Und das hieß, die Flüchtlinge auf verschiedene Länder zu verteilen. Zweiundreißig Staaten nahmen teil, darunter Brasilien - notgedrungen. Der Schein musste erneut gewahrt werden. Das positive 
Image sollte auf keinen Fall leiden, und das obwohl die Ziele natürlich mit der brasilianischen Politik überhaupt nicht vereinbar waren. Der brasilianische Außenminister empfahl seiner Delegation, das geheime Rundschreiben 1.127 stets bei ihren Entscheidungen mit zu berücksichtigen. Darauf weist das Buch ausdrücklich hin, auch darauf, dass den meisten Juden, denen trotz allem mit einem Touristenvisum die Einreise gelungen war, keine Daueraufenthalts- und Arbeitsgenehmigung gegeben wurde. (ebd.: 87; 91) Die Ausweisung schwebte damit als Damokles-Schwert ständig über ihnen.

Dass Antisemitismus, Rassismus und Diskriminierung die Lebensthemen der Paulistaner Historikerin Maria Luiza Tucci Carnerio sind, zeigt ihr Buch eindrucksvoll. Sie ist Professorin für Sozialgeschichte an der Universität Sao Paulo und Leiterin des „Laboratório de Estudos sobre Etnicidade, Racismo e Discriminacao“. Ein großes Projekt dieses Laboratoriums ist die Erarbeitung eines virtuellen Archivs über den Holocaust, mit abrufbarer Website zur jüdischen Einwanderung (Vgl. www.arqshoah.com.br ). Tucci CARNEIRO hat besonders nach dem Wendepunkt in der brasilianischen Geschichtsschreibung, der Öffnung des historischen Archivs des Außenministeriums Itamaraty, und des Archivs der politischen Polizei in Sao Paulo 1995 zur jüdischen Einwanderung publiziert. Nach Veröffentlichung ihres Buches Brasilien, Fluchtpunkt in den Tropen [Brasil, um refúgio nos trópicos, 1996] folgte dann 2010 Cidadão do mundo, ein Werk, das auf ihrer Habilitationsschrift von 2001 beruht und das nun auf Deutsch vorliegt. Arbeiten zum Antisemitismus, zu den Menschenrechten, zur ethnischen und politischen Intoleranz sowie zur Zensur runden ihr Werk ab.

Dass Maria Luiza Tucci Carneiro die auf Schein beruhende brasilianische Politik schonungslos aufklärt und belegt, wundert bei ihrem Forschungsschwerpunkt nicht, und ist das große Verdienst ihres umfangreichen Buches. Die restriktive Einwanderungspolitik verschloss mit ihren geheimen Rundschreiben die Türen Brasiliens für viele Juden. Die Begründung der brasilianischen Regierung war kurz und klar: Juden wurden als Ausbeuter, Verschwörer und Kommunisten gesehen. An Unterstützern fehlte es nicht, auch darauf legt Tucci Carneiro ihren Finger: Die katholische Kirche billigte diese Politik ebenso wie die Streitkräfte oder die zuständigen Ministerien (Vgl. CARnEIRo 2014: 416). Das erstaunt bei der Kirche nicht, die ja über die „Rattenlinie“ und den Vatikan zahllosen Nazis, auch Nazimördern, zur Flucht nach 
Lateinamerika verholfen hat. ${ }^{3}$ Zum Beispiel Walter Rauff, Chef der Vergasungswagen, in denen 180.000 Juden ermordet wurden, der über Ecuador nach Chile floh. Dass dieser Mann dann von 1958 bis 1962 für den westdeutschen Nachrichtendienst gearbeitet und aus Lateinamerika berichtet hat, ist einer der vielen Skandale der Nachkriegszeit in der ehemaligen Bundesrepublik. (Vgl. CARNEIRO 2014: 97).

Wie schade, dass Stefan Zweig dann 1941 Brasilien noch einmal als „Land der Zukunft“ ohne Rassenprobleme feierte. Dieses Land schickte Olga Benario in den Tod und verhinderte auf Grund des Rassismus seiner Regierung die Einreise von vielen anderen Juden. Der „Ritter der Hoffnung“, Luiz Carlos Prestes, erfuhr erst nach seiner großen Rede im Paulistaner Stadion „Pacaembu“ im Juli 1945, dass seine Frau Olga ermordet worden war. Seine Tochter Anita lernte er auch erst damals kennen. Sie ist davon überzeugt: „Die jungen Leute heute können sich überhaupt nicht vorstellen, dass zum Beispiel Folter damals eine Normalität war. Die nach der Diktatur geborene Generation ist leider ziemlich unpolitisch. Sie weiß wenig über die Geschichte, und die Medien tun das Ihre dazu, damit das auch so bleibt“"4

Eine Einschätzung, die auch für Deutschland gilt, ein Land, dem der ehemalige Bundeskanzler Willy Brandt eine zweite Bücherverbrennung im Sinne der Nichtnutzung der Erfahrungen bescheinigte.

Ein mutiges Buch, ein fleißiges und umfassendes Buch, das zahllose neue Quellen erschlossen hat. Dass die Mauer des Schweigens in Brasilien brechen konnte und damit einen Wendepunkt darstellt. Deshalb ist es so wichtig für deutsche Historiker, aber auch für das allgemeine Publikum. Stefan Zweigs Lobgesang und Schwärmereien aus dem Jahre 1941 sollten nicht das letzte Wort behalten.

Hier und da leidet allerdings die Lesbarkeit etwas an dieser Fülle. Das Buch behandelt seinen Stoff in sieben Kapiteln über 477 Seiten und geht dabei beispielsweise auch auf Themen wie die Flucht der Gruppe Görgen, die landwirtschaftliche Kolonie Rolandia in Paraná, ein Rettungspunkt für viele Flüchtlinge, oder auf die Palästinafrage im Jahre 1947 ein. Zweiundzwanzig Minibiografien der wichtigsten Protagonisten wie

\footnotetext{
${ }^{3}$ Vgl. GIEFER, Rena; GIEFER, Thomas. Die Rattenlinie. Fluchtwege der Nazis. Eine Dokumentation. Frankfurt am Main 2/1992.

4 Äußerung von Anita Leocadia Prestes während der Vorstellung unseres Sammelbandes Não olhe nos olhos do inimigo, 1996 in São Paulo, Brasilien.
} 
Strauss, D. - Entlarvung des Antisemitismus

Vargas oder Dutra, eine ausführliche Bibliografie, chronologisch geordnete Archivquellen und ein Personen-Index schließen das Werk ab.

Da hätte man sich grundsätzlich etwas mehr Straffheit gewünscht. Bedauerlich, dass dieses wichtige Buch so teuer ist. 49,90 Euro werden wohl nur von Institutionen bezahlt, kaum von dem Einzelleser, zumal die Klebebindung auch sehr schnell nachgibt.

Recebido em 30/09/2014 aprovado em 20/10/2014 